
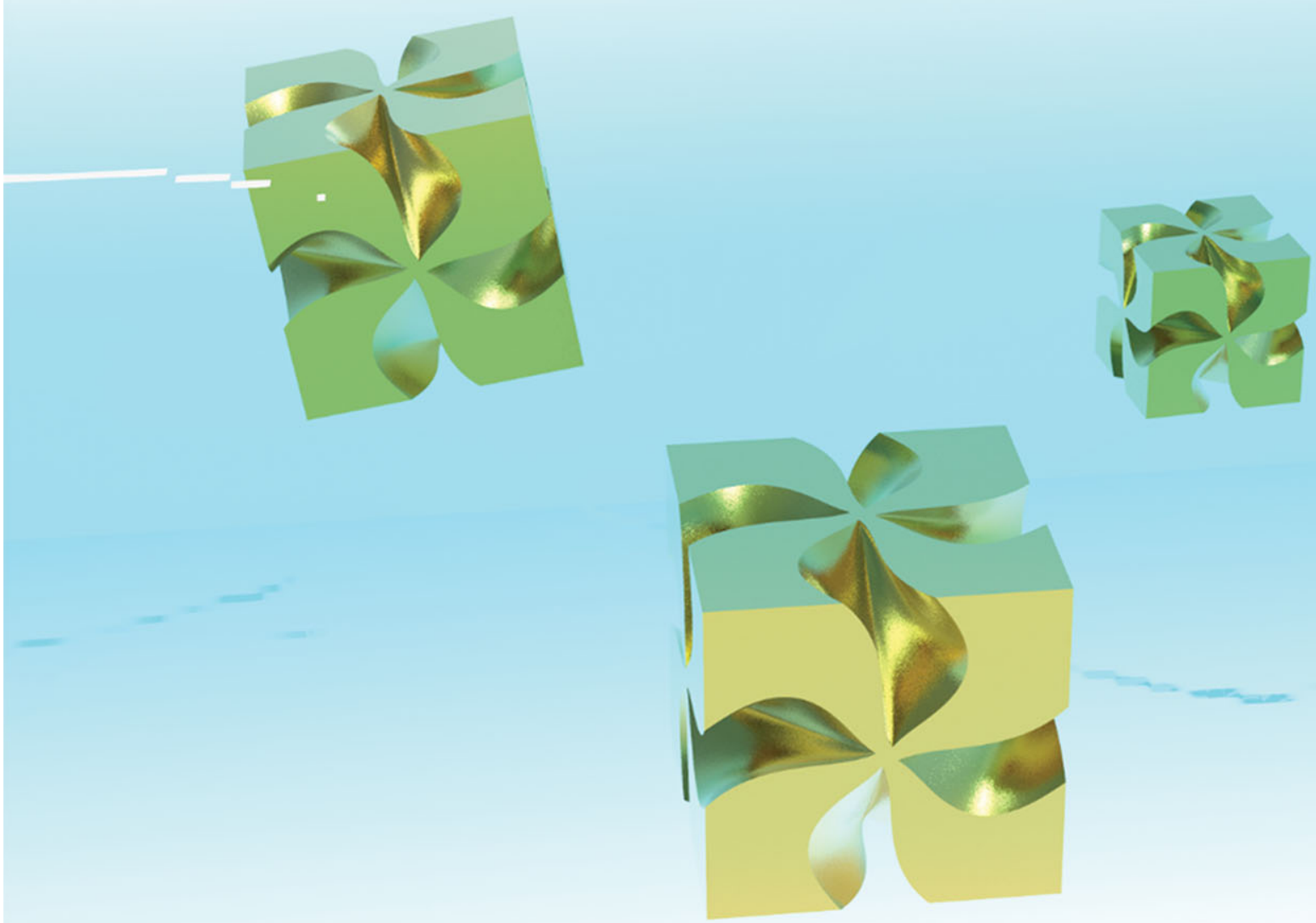

Showcasing research from Professor Ki Tae Nam's laboratory, Department of Materials Science and Engineering, Seoul National University, Seoul, Republic of Korea.

Chirality control of inorganic materials and metals by peptides or amino acids

Inorganic materials and metals can develop chiral morphologies that are precisely controlled by the amino acids and peptides. The chirality of biomolecules can be transferred to the inorganic materials due to the enantioselective interactions between inorganic surfaces and amino acids/peptides. Herein, we review the reported literature from the fundamental aspects of chirality in inorganic and metal crystals. We concentrate our attention on the exceptional roles of amino acids/peptides on the interaction between organic molecules and inorganic surfaces, the reconstruction of the local atomic conformation, and the macroscopic evolution of chiral morphology.
As featured in:

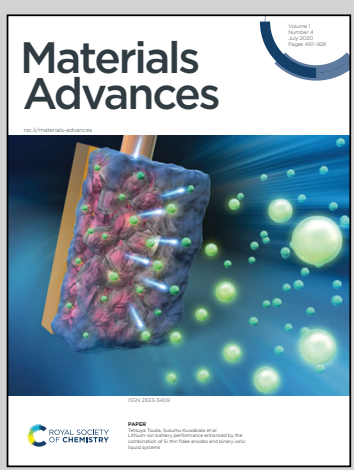

See Ki Tae Nam et al., Mater. Adv., 2020, 1, 512. 
Check for updates

Cite this: Mater. Adv., 2020 1,512

Received 24th March 2020 Accepted 5th May 2020

DOI: 10.1039/d0ma00125b

rsc.li/materials-advances

\title{
Chirality control of inorganic materials and metals by peptides or amino acids
}

\author{
Hyeohn Kim, $\dagger$ Sang Won Im, $\dagger$ Ryeong Myeong Kim, $\dagger$ Nam Heon Cho, \\ Hye-Eun Lee, Hyo-Yong Ahn and Ki Tae Nam (iD*
}

\begin{abstract}
Chirality exists everywhere in nature and may be one of the most important features in biological systems. The chirality of amino acid molecules is transferred to the peptide sequences, determining the secondary and further three-dimensional structures. As a result, even the macroscopic chirality observed in many living features can be controlled by the peptide sequence. Interestingly, recent studies have shown that achiral inorganic materials and metals, according to the crystallographic point group, can develop chiral morphologies that are precisely controlled by the amino acids and peptides. As a result, strong chiral optical responses can be generated even at visible wavelengths. In this review, we have highlighted recent pioneering examples to show the enantioselective interactions between inorganic materials/metals and amino acids/peptides and discussed the underlying mechanisms.
\end{abstract}

\section{Introduction}

Chirality, one of the key features in living organisms, can be found from the molecular level, such as in DNA and peptide structures, to the macroscopic level, such as in seashells, snails, and even flowers. Chirality means mirror symmetry, the molecular understanding of which was first conceptualized by Louis Pasteur in $1848 .^{1}$ This significant paper was published in Annales de Chimie et de Physique and was translated as "the relations that may exist between the crystalline form, the chemical composition and the direction of the polarization".

Department of Materials Science and Engineering, Seoul National University, Seoul, Republic of Korea.E-mail: nkitae@snu.ac.kr

$\dagger$ Equally contributed to this work.
As we may notice from the title, this work investigates how optical rotation properties can be different in two different crystals of tartaric acid, one from natural wine and another from organic synthesis. This discovery provides new insights to recognize molecular chirality and to link its handedness with the measured physical properties, more importantly inspiring new fundamental sciences on the symmetry problem.

Molecules with opposite chirality exhibit completely different properties despite having identical compositions. For example, $R$-thalidomide is an effective sleeping supplement for pregnant women, while the molecule with opposite chirality causes malformation of limbs in babies. Additionally, $R$-limonene smells like oranges, whereas the opposite enantiomer possesses the characteristic smell of lemons. This is due to receptors or sensors in our human body being intrinsically

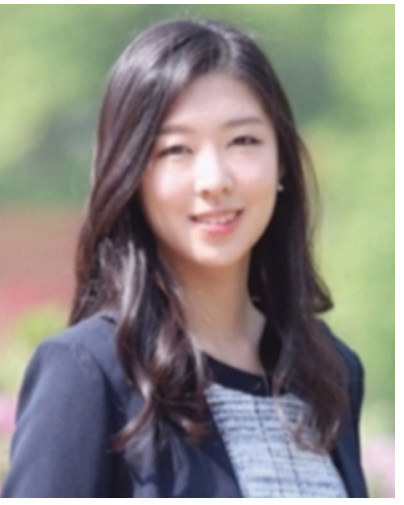

Hyeohn Kim
Hyeohn Kim received her BS and MS degrees from Yonsei University. She is currently pursuing her PhD in the Department of Material Science and Engineering, Seoul National University, working under the supervision of Prof. Ki Tae Nam.

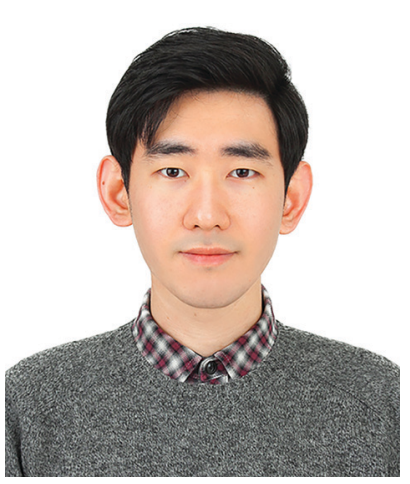

Sang Won Im
Sang Won Im received his BS degree from Seoul National University. $\mathrm{He}$ is currently pursuing his $\mathrm{PhD}$ in the Department of Material Science and Engineering, Seoul National University, working under the supervision of Prof. Ki Tae Nam. 
chiral, and the opposite recognition can result in different signaling and outcomes. Therefore, in organic synthesis, chiral-selective synthesis and separation have become more significant, especially in the pharmaceutical or nutrient industry. Beyond molecular and organic chirality, a lot of new opportunities exist in controlling the chirality of electrons, which include the spin, and the chirality of the electromagnetic wave, which is polarization. These scientific impacts can be extended to spintronics, quantum devices and metamaterials, resulting in new applications. For this expansion from molecular chirality to other physical phenomena, the fundamental challenges are (1) how chiral-selective interaction can be achieved at different scales between dissimilar media, (2) how chirality can be transferred from molecules to macroscopic features, and (3) how chiral matter can manipulate photons and spin, having the wave-particle duality.

The translation of our understanding of molecular chirality to the larger scale synthetic technology is difficult but it seems possible if we watch many beautiful examples in biological systems. Biological systems utilize the interface between proteins and structural materials such as calcium carbonate and chitin to obtain macroscopic chiral structures. Butterfly wings, exoskeletons of insects, or seashells can serve as model systems of self-assembled biological chiral structures. Snails, gastropods, have a spirally coiled shell, showing a chiral preference for either right- or left-handed spirals. ${ }^{2}$ The chitin distribution in the wings of the Callophrys rubi butterfly can be described as a chiral gyroid structure. ${ }^{3}$ An important lesson we can learn is that specific proteins can direct the chiral assembly and thus, the chirality of the proteins is transferred macroscopically.

Herein, we review the reported literature from the fundamental aspects of chirality in inorganic and metal crystals. We concentrate our attention on the exceptional roles of amino acids and peptides on (1) the interaction between organic molecules and inorganic surfaces, (2) the reconstruction of the local atomic conformation, and (3) the macroscopic evolution of chiral morphology.

\section{Chirality in inorganic and metal crystals}

The chirality in inorganic materials and metals has been less harnessed as compared to the chirality in organic molecules. While chiral centers can be clearly used as a reference points to distinguish the chirality in organic molecules, the determination of chirality in inorganic materials and metals requires additional consideration of the relative positions of atomic coordination and planes. Therefore, the identification of chiral components in inorganic and metallic structures is less straightforward and more difficult as compared to organic molecules. However, intriguing properties of crystal chirality have gained attention even before the discovery of the chirality of organic molecules. Before the discovery by the Pasteur, the different rotations of linearly polarized light were characterized with quartz crystals in $1811 .{ }^{4}$ The study extended from observing and exploiting the naturally occurring chiral crystals to controlling and inducing chirality on crystals, even on those that are intrinsically achiral. Enantioselective interactions between crystals and chiral organic molecules have been the key aspect in this field.

Some inorganic crystals have bulk chirality due to helical arrangements along certain crystallographic directions. ${ }^{5-9}$ These crystals show a lack of symmetry operations that induce achiral structures, namely mirrors, glide planes, an inversion center, and a roto-inversion operator. Among 32 crystallographic point groups, only 11 point groups meet this requirement. The most common example would be quartz (space group $P 3_{1} 21$ or $P 3_{2} 21$ ), in which the corner-linked $\mathrm{SiO}_{4}$ tetrahedron is the basic unit of helices. Similarly, other inorganic materials such as $\alpha-\mathrm{AlPO}_{4}$ and $\alpha-\mathrm{GaPO}_{4}$ having the tetrahedral phosphate network also show chirality. Another example is cinnabar $\left(\alpha-\mathrm{HgS}\right.$, space group $\left.P 3_{2} 21\right)$, whose crystal structure has helices of repeated $\mathrm{Hg}$ and $\mathrm{S}$ atoms along the $z$-axis, as shown in Fig. 1A. ${ }^{6}$ Depending on the orientation of the helical atomic arrangements, chiral crystals have left- and right-handed

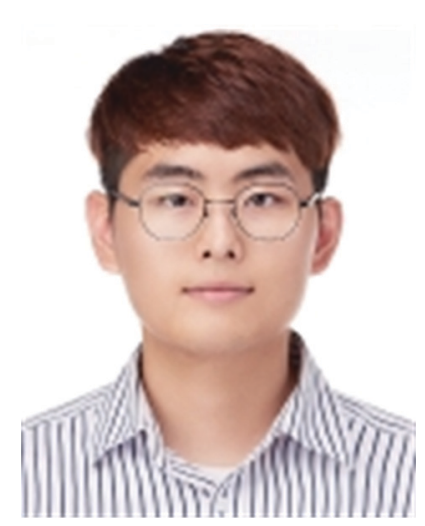

Ryeong-Myeong Kim
Ryeong-Myeong Kim received his $B S$ degree from Ulsan National Institute of Science and Technology. He is currently pursuing his $P h D$ in the Department of Material Science and Engineering, Seoul National University, working under the supervision of Prof. Ki Tae Nam.

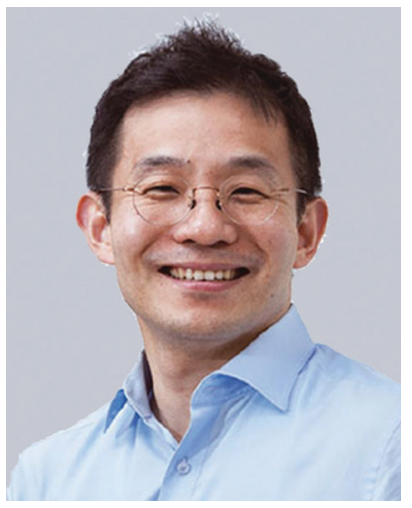

Ki Tae Nam
Ki Tae Nam received his $P h D$ from Massachusetts Institute of Technology in 2007. He worked as a postdoctoral fellow in the Molecular Foundry, Lawrence Berkeley National Laboratory until 2010. He is currently a professor in the Department of Materials Science and Engineering at Seoul National University. His research is focused on the interface between peptides and inorganic materials and peptide selfassembly. His research group can be found at wuw.nkitae.org. 

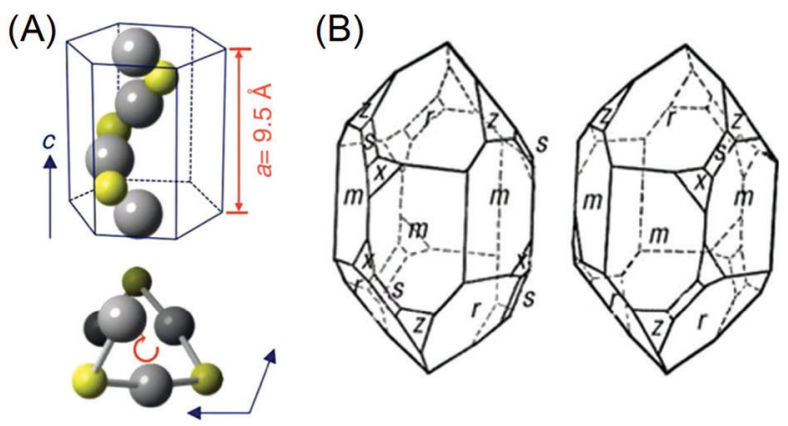

(C)
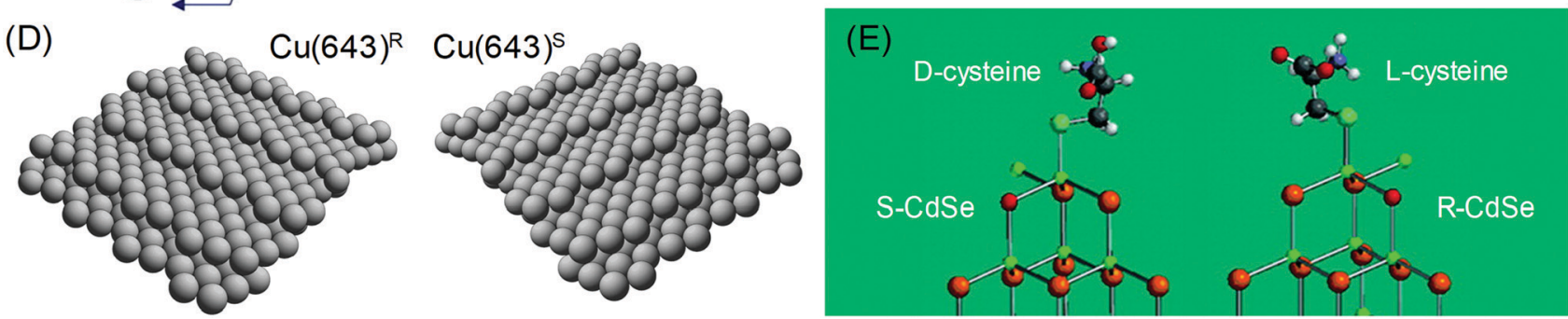

Fig. 1 Chirality of the crystal structure and surface: (A) The helical arrangement of $\mathrm{Hg}$ and $\mathrm{S}$ atoms in the crystal structure of cinnabar ( $\alpha-\mathrm{HgS})$. (B) Leftand right-handed variants of quartz $\left(\mathrm{SiO}_{2}\right)$ crystal. Common crystal surfaces are noted as $m(100), r(101), s(111), x$ (511). (C) Trigonal scalenohedral crystal structure of calcite $\left(\mathrm{CaCO}_{3}\right)$ and the surface $\mathrm{CaO}_{6}$ octahedra structure of the $\left(3 \overline{1} \overline{2}\right.$ face (left)) and the (21̄ face (right)). (D) Models of the ideal $\mathrm{Cu}(643)^{R}$ and $\mathrm{Cu}(643)^{\mathrm{S}}$ surfaces. The structure is based on a (111) terrace, separated by a (100) step edge and (110) kink. (E) The lowest energy configurations of (D-cysteine, S-CdTe) and (L-cysteine, R-CdTe). Atoms are noted as follows: Cd (green), Te (brown), O (red), H (light gray), S (cyan), C (gray). (A) Reprinted with permission. ${ }^{6}$ Copyright (C) 2019, Springer Nature. (B), (C) Reprinted with permission. ${ }^{10}$ Copyright (C) 2019, Springer Nature. (D) Reprinted with permission. ${ }^{8}$ Copyright (C) 2019, Springer Nature. (E) Adapted with permission. ${ }^{17}$ Copyright (C) 2010, American Chemical Society.

variants, which occur in approximately equal proportions in nature. The handedness of these crystals is often distinguished by their opposite optical rotation properties or exposed chiral crystal faces. For example, polarized light passing through the $c$-axis is rotated in the opposite direction depending on the handedness of the quartz; counter-clockwise (or levorotary) in left-handed quartz and clockwise (or dextrorotary) in right-handed quartz. ${ }^{9}$ However, this relationship between the handedness of the crystal and the optical rotatory direction does not apply to all chiral crystals but often shows the opposite relationship. Another interesting characteristic of crystals with bulk chirality is that their surface structures also contain chirality. While the atomic arrangements of all crystal faces of quartz are chiral, some crystal faces such as the (111) and (511) faces (Fig. 1B, denoted as $x$ and $s$ respectively) show observable differences in orientation, becoming distinguishable features of left- and right-handed crystals. ${ }^{7}$

Chiral surfaces of achiral crystals may seem counterintuitive but surface chirality is not exclusive to inorganic or metal crystals with bulk chirality. Surface chirality can occur in achiral inorganic crystals with any crystal faces that a lack perpendicular mirror symmetry operator. ${ }^{8}$ This condition is much easier than bulk chirality and is met by common crystal faces of many achiral inorganic crystals. For example, calcite $\left(\mathrm{CaCO}_{3}\right.$, rhombohedral space group $\left.R \overline{3} c\right)$, which is a prevalent mineral found as limestone, marble, or biominerals, has been reported for its enantiomorphic crystal faces. ${ }^{10}$ The $\{21 \overline{3} 1\}$ faces of the common trigonal scalenohedral form of calcite crystals have chirality due to the glide plane of the corner-linked $\mathrm{CaO}_{6}$ octahedra (Fig. 1C). Other silica minerals that are abundant in the earth's crust, such as alkali feldspar $\left(\mathrm{KAlSi}_{3} \mathrm{O}_{8}\right.$, monoclinic space group $C 2 / m$ and $\mathrm{NaAlSi}_{3} \mathrm{O}_{8}$, triclinic space group $\left.C \overline{1}\right)$ and clinopyroxenes ((Ca, $\mathrm{Mg}, \mathrm{Fe}) \mathrm{SiO}_{3}$, monoclinic space group $\left.\mathrm{C2} / \mathrm{c}\right)$, also show chirality in their common crystal faces. ${ }^{7}$ Similarly, surface chirality exists even for highly symmetric metal crystals. Gellman $^{11}$ and Attard ${ }^{12}$ conducted a pioneering work by proposing an atomic kink site at high-Miller-index surfaces as the intersection of a low-Miller-index $\{111\}$ terrace, $\{100\}$ step and $\{110\}$ step (Fig. 1D). The handedness of the kink site can be defined as the ordering of low-index crystal planes where $\{111\} \rightarrow\{100\} \rightarrow\{110\}$ is oriented as clockwise and anticlockwise rotation, which can be expressed as $\mathrm{R}$ and $\mathrm{S}$, respectively.

The interesting property of the chiral surfaces of inorganic and metal crystals is enantioselective interactions with chiral organic molecules. ${ }^{13-19}$ Depending on the handedness of the crystal surfaces, chiral molecules selectively adsorb or bind to the surface. Research has been conducted on the adsorption of chiral molecules on well-defined single-crystalline high-Millerindex metal surfaces. Temperature-programmed desorption (TPD) confirmed the enantioselective adsorption of small chiral molecules on chiral metal surfaces, such as 2-butanol on $\mathrm{Ag}(643)$ and propylene oxide and 3-methylcyclohexanone on $\mathrm{Cu}(643){ }^{10}$ Moreover, enantioselective interactions between molecules and crystal surface can even induce chirality in achiral faces. It has been reported that the adsorption of a chiral molecule on an achiral metal surface induced the reconstruction to a chiral highMiller-index facet. For example, the adsorption of $\mathrm{L}$-lysine on the $\mathrm{Cu}(100)$ surface caused the formation of the $(3,1,17)^{\mathrm{R}}$ facet. $^{16}$ Also, 2,5,8,11,14,17-hexa-(tert-butyl)decacyclene on the $\mathrm{Cu}(110)$ surface generated chiral kink sites that are similar to the ones 
observed on $\mathrm{Cu}(643){ }^{8}$ For inorganic crystals, induced chirality on the CdTe nanocrystal by $\mathrm{L}^{-}$and $\mathrm{D}^{-}$cysteine has been observed. ${ }^{17}$ The Kotov group proposed that after the binding of cysteine, the exposed $\mathrm{Cd}$ atom can have 4 different bonds, just like the chiral centers of organic molecules. DFT calculations showed that $\mathrm{L}^{-}$and $\mathrm{D}$-cysteine selectively stabilized $R$ - and $S$-type chiral Cd centers (Fig. 1E), showing chirality-dependent interactions between the surface of the inorganic crystal and the chiral molecule.

\section{Chiral-optical response from peptide- and amino acid-attached inorganic nanoparticles}

The origins of the chiral properties of inorganic and metal materials are not restricted to the chirality of crystal structure or surface. By interaction with surrounding chiral organic molecules, even achiral crystals can exhibit chiral properties in the optically active range of metals. This kind of induced chirality often emerges from the electronic coupling between organic molecules and the local electric field of metal or inorganic crystals. $^{20,21}$ Metal nanoparticles with localized surface plasmon or optical inorganic nanocrystals with excitonic optoelectronic properties are often selected as materials for coupling with chiral molecules. The most significant results are chiroptical properties, represented by circular dichroism (CD) and optical rotatory dispersion (ORD), which are enantioselective absorption for circularly polarized light and optical rotation of linear polarized light, respectively. Induced chirality on plasmonic metal nanomaterials enables the amplification and detection of molecular circular dichroism signals in the visible range. The easy and facile detection of molecular chirality is critical in industrial sectors where bimolecular enantiomers exhibit different effects, such as in the medical, pharmaceutical, catalytic and chemical industries. Also, chiral absorption and emission of optical nanomaterials have been researched as possible applications for metamaterials that exhibit extraordinary properties that are not observed in common bulk materials. ${ }^{22-25}$

Localized Surface Plasmon Resonance (LSPR) is the oscillation of electrons near the plasmonic nanoparticle surface when they are illuminated with light. These resonating fields are concentrated at the surface of metal nanoparticles and decay exponentially with increasing distance from the surface. As such, researchers focus on the surface of plasmonic nanoparticles to transfer local electric field energy from plasmonic nanoparticles to organic molecules. ${ }^{9,26-29}$ Gautier et al. showed that simple gold nanoclusters can generate opposite chiroptical signals upon the attachment of ligands to their surfaces. Inversion of the CD signal was observed with the exchange of the conjugated ligand from the $\mathrm{L}$ form to the $\mathrm{D}$ form $(R$ form to $S$ form) (Fig. 2A). ${ }^{30}$ Govorov et al. theoretically supplemented the experimental results, which showed the utilization of plasmon resonance for amplifying chiroptical signals through interacting with chiral molecules. ${ }^{26}$ Two possible explanations were proposed: plasmon-enhanced molecular dipole influencing the CD signals of chiral molecules, and molecular dipole-induced chiral currents inside the metal nanocrystal. These two factors combine to create the optical responses of metal-molecule complexes.

Gregorio et al. showed that silver nanocubes with glutathione ligands induced CD signals in their plasmon resonance

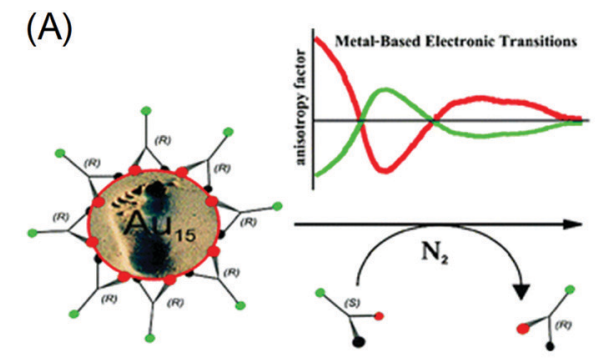

(C)

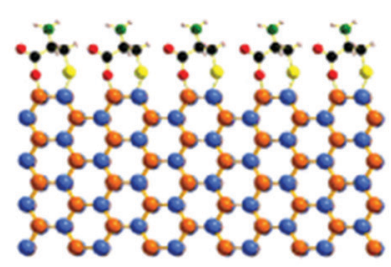

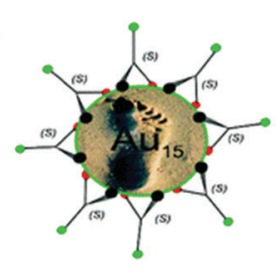

(B)
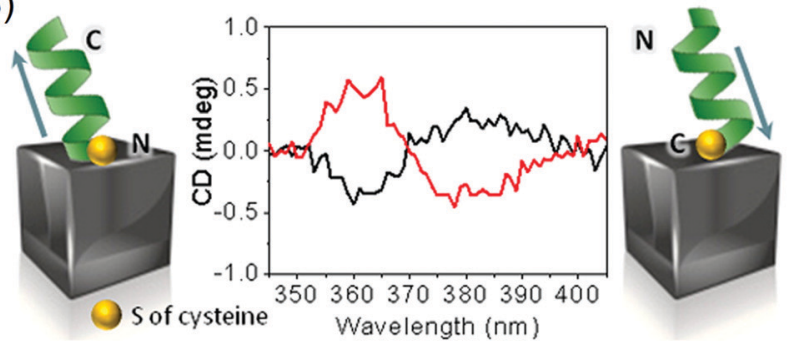

(D)

Zincblende CdSe

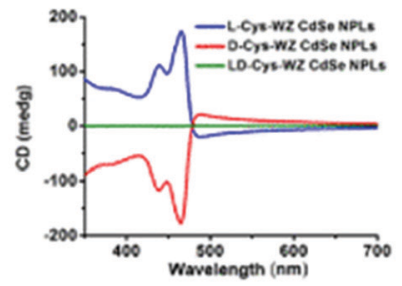

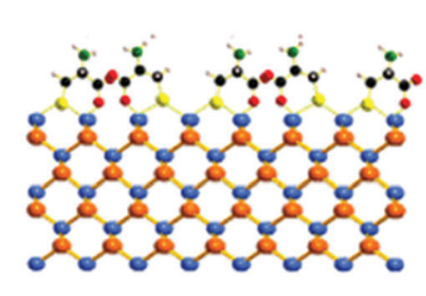

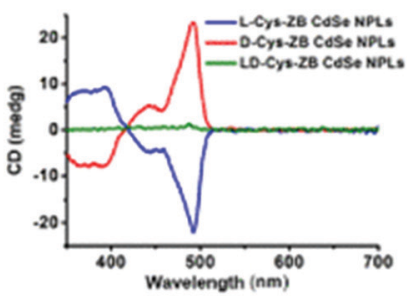

Fig. 2 Chiral-optical response from peptide and amino acid-conjugated plasmonic nanoparticles (induced chirality). (A) Inversion of the CD signal of chiral thiol-conjugated gold nanoparticles. (B) Helical peptide-conjugated silver nanocubes and their CD signals for the opposite helicity of peptides. (C) The surface of wurtzite CdSe nanoplatelets conjugated with cysteine molecules and their CD signal for different enantiomers. (D) The surface of zinc blende CdSe nanoplatelets conjugated with cysteine molecules and their CD signals for different enantiomers (right). (A) Reprinted with permission. ${ }^{30}$ Copyright (C) 2008, American Chemical Society. (B) Reprinted with permission. ${ }^{32}$ Copyright (C) 2016, American Chemical Society. (C), (D) Adapted with permission. ${ }^{34}$ Copyright 2018, American Chemical Society. 
range. The origin of the CD signal was confirmed through the oxidation reaction of the thiol group attached to the silver nanocube surface; the detachment of the ligand eliminated the existing chiroptical response. ${ }^{31}$ Levi-belenkova et al. also utilized silver nanocubes to induce a chiroptical signal using polyproline-2-based helical peptides as the chirality inducer. Induction of the chiroptical signal in the silver nanocubepeptide hybrid structure was proposed to have originated from multipole mode interactions between organic molecules and the inorganic silver nanocube surfaces (Fig. 2B). ${ }^{32}$

The induced chirality of the inorganic crystal has been studied for quantum dots, rods, or wells to achieve various chiroptical properties. Extensive work has been done on II-VI semiconductor nanomaterials such as CdS and CdSe. ${ }^{33-42}$ Various reports have shown that circular dichroism was observed when chiral molecules, typically cysteine, ${ }^{33,35-37,41}$ were attached to achiral crystals. Theoretically, experimental and calculated results showed that the circular dichroism can be understood as the result of electronic coupling between chiral molecules and dielectric nanocrystals rather than structural chirality. Govorov et al. theoretically predicted that the circular dichroism resulted from the coupling between achiral dielectric nanoparticles and chiral molecules. ${ }^{26}$ Experimental results showed that circular dichroism can be induced by controlling the chirality of the attached ligands on achiral quantum dots. Xiaoqing Gao et al. also reported induced chirality on CdSe nanoplatelets using $\mathrm{L}^{-}$and D-cysteine ligands. ${ }^{34}$ Opposite circular dichroism was observed for $\mathrm{L}^{-}$and D-cysteine, along with distinguishable spectra that were dependent on the crystal structure. When L-cysteine was used as the ligand, wurtzite showed three upward circular dichroism peaks in the visible range (Fig. 2C), while zinc blende showed one downward and two upward peaks (Fig. 2D). The experimental results were further understood through DFT calculations on the non-degenerate coupled-oscillator model, which describe the coupling of the electronic transition of CdSe nanoplatelets and cysteine. The divergent circular dichroism responses dependent on the CdSe crystal structure resulted from differences in the configurations of the surface cysteine molecules on wurtzite and zinc blende CdSe and several couplings of electron transitions among the various chromophores.

\section{Chirality generated by local distortion}

Another interesting strategy for inducing chiroptical signals in inorganic materials is through the distortion of the local atomic conformation on the surface of the inorganic material through organic-inorganic interactions. Chirality from atomic distortion is different from organic molecule-induced chiroptical signals in that their origin of chirality is directly from the material itself. In this part, representative theoretical and experimental backgrounds and examples of chirality emergence by local distortion will be reviewed.
Plasmonic materials have often been chosen as ideal materials for observing chiroptical response. ${ }^{27,28,43,44}$ Schaaff et al. observed the chiroptical response generated through the deposition of monolayers of glutathione (GSH) molecules on gold clusters in 1998 and proposed that the GSH molecule induced chiral adsorption patterns onto the gold cluster surface. ${ }^{44}$ Similarly, Jadzinsky et al. synthesized and analyzed the atomic structure of $p$-mercaptobenzoic acid ( $p$-MBA)-deposited gold nanoparticles through powder X-ray diffraction analysis. $p$-MBA-capped gold nanoparticles showed the chiral arrangement of the atomic coordination within a unit cell scale (Fig. 3A). ${ }^{43}$

In addition to chirality transfer from organic to plasmonic materials through local distortion, methodologies and strategies for applying these concepts to semiconducting materials were enthusiastically explored due to the excellent optical and electrical properties of semiconducting materials ${ }^{17,45-47}$ Molony et al. synthesized cadmium sulfide (CdS) nanoparticles by attaching L \& D penicillamine molecules onto the surface of CdS nanoparticles. The adsorbed penicillamine induced different chiralities in the CdS nanoparticles and their optical activities were determined through CD measurements. Totally inverted signals for L \& D penicillamine-treated CdS nanoparticles were observed. Also, CdS nanoparticles treated with racemic penicillamine molecules showed 0 circular dichroism signals. The induced chirality of the inorganic nanomaterials through local distortion was shown to be sustained by the attachment of any type of ligand, and even the presence of achiral ligands could stabilize the distorted surface. ${ }^{45}$ In a subsequent study, Elliott et al. took the previous study one step further through the DFT simulation of synthesized CdS nanoparticles with L, D \& rac penicillamine. The simulation results revealed that the introduced penicillamine ligands were strongly bound to the nanoparticle surface via $\mathrm{N}$ - and $\mathrm{S}-\mathrm{Cd}$ bonding and the additional bonding of carboxylates to nearby Cd-induced chirality in the nanoparticles.

Similarly, the Nakashima group synthesized thiolated ligand adsorbed CdTe nanoparticles to cause local distortion on the CdTe nanoparticle surfaces (Fig. 3B). After observing the optical activity of CdTe nanoparticles, ligand exchange from chiral molecules to achiral ones retained the chiroptical activity of the pre-synthesized CdTe nanoparticles. ${ }^{46}$ Zhou et al. specifically analyzed chirality transfer through the local distortion of the surface through the inspection of the atomic configuration and structure of the inorganic material surface. Through experimental and computation analysis, CdTe nanomaterials covered with L \& D cysteine molecules showed chiroptical responses through atomic distortion and the specific structure was later thermodynamically predicted through calculations. The calculated results corresponded to the opposite atomic conformation for L \& D enantiomers. This finding facilitated the understanding of the local chiral distortion from chiral organic molecules, which provided strong evidence for chirality generation through atomic distortion in inorganic nanomaterials. Furthermore, this work provided grounds for further research on the relationship between nanostructures or atomic configurations and the degree of optical activity. ${ }^{17}$ 

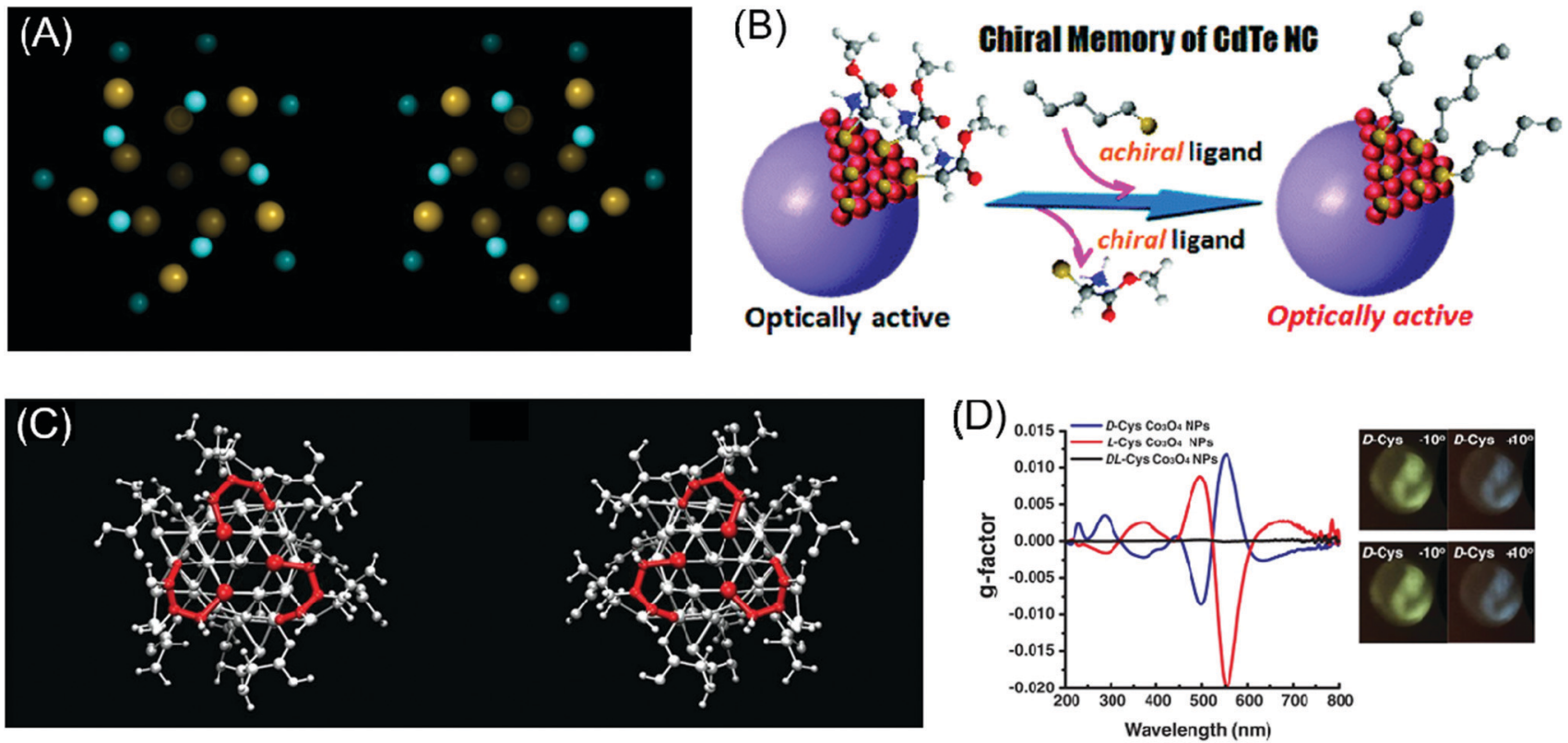

Fig. 3 Chirality emergence by local distortion. (A) The view of thiol-monolayer-protected gold nanoparticles along their cluster axis for two enantiomeric particles. (B) Scheme of the chiral memory effect in semi-conducting CdTe nanoclusters. (C) The configurations of D-cysteine (left) and $L$-cysteine (right)-functionalized $\mathrm{CO}_{3} \mathrm{O}_{4}$ nanoparticles depict different chirality generations for different enantiomers. (D) The $g$-factor of $\mathrm{L}$, $\mathrm{D}$ and racemic cysteine-treated $\mathrm{Co}_{3} \mathrm{O}_{4} \mathrm{NPs}$ (left), visualization of the ability to rotate the polarization axis of $\mathrm{CO}_{3} \mathrm{O}_{4} \mathrm{NPs}$. The analyzer rotates from $-10^{\circ}$ (counterclockwise) to $10^{\circ}$ (clockwise) (right). (A) Reprinted with permission. ${ }^{43}$ Copyright (C) 2007, American Association for the Advancement of Science. (B) Reprinted with permission. ${ }^{46}$ Copyright (C) 2009, American Chemical Society. (C) and (D) Reprinted with permission. ${ }^{49}$ Copyright (C) 2018, American Association for the Advancement of Science.

Jiang et al. adopted these explanations to control and enhance the optical activity of nanomaterials by using two different types of chiral molecules. In their study, chiral ceramic materials (tungsten oxide hydrate nanoparticles) with two different amino acids, proline and aspartic acid, were synthesized with the expectation of the generation of multiple degrees of local distortion when attached to the surface of ceramic nanoparticles. Depending on the intrinsic chirality of the molecules adsorbed on the inorganic nanomaterial surfaces, the degrees of local distortion and optical activity were different. ${ }^{48}$

In the case of magnetic materials, chirality through atomic configuration was generated with a higher dimension of controllability. Conventionally, after fabrication or synthesis, material properties, especially chirality, were a permanent feature of nanomaterials unless irreversible chemical treatment was applied. With an external magnetic field to induce local distortion in inorganic materials, the controllability of handedness on the atomic scale was allowed. Representatively, Yeom et al. tested L \& D cysteine-induced chiral paramagnetic cobalt oxide nanoparticles under a magnetic field (Fig. 3C). These cobalt oxide nanoparticles originally showed strong chiroptical responses while under exposure to an external and non-invasive magnetic field; the local atomic configuration was controlled to modulate the chiroptical signal (Fig. 3D). Tuning of the chirality through the external magnetic field showed the versatile potential of chiral nanomaterials for application in industry. ${ }^{49}$

In this section, representative results and recent advances in chirality transfer from organic molecules through the local distortion of inorganic materials were reviewed. In the next section, the evolution of the macroscopic morphology change in nanomaterials induced from local distortion and the relationship between macro- and nanostructures and their chiroptical responses will be reviewed.

\section{Chiral morphology}

The exceptional abilities of biological molecules to induce the reconstruction of inorganic interfaces enable macroscopic changes in the final nanomaterial morphology. Precisely designed 3D chiral morphologies of inorganic nanostructures can be obtained through the encoding of biological molecules, thereby maximizing the chiroptic activity between RCP and LCP. ${ }^{25,50}$ In this section, we discuss the evolution of the chiral morphology and chiroptical response at the single nanostructure level and macroscopically hierarchical chiral structures composed of single chiral units in semiconducting materials. In addition, we address the chiral morphology evolution in plasmonic materials based on the strong interactions of amino acids and peptide-based materials with metal surfaces. Furthermore, we would like to highlight single plasmonic chiral nano-morphology with exceptional chiroptical activity.

\subsection{Chiral morphology evolution in inorganic materials}

The macroscopic chirality of inorganic materials could evolve from the enantioselective adsorption of chiral molecules onto 
their crystal planes. ${ }^{51,52}$ In particular, materials with crystallographic chirality show advantages in efficiently tailoring chiral morphology and optical properties. The adsorption

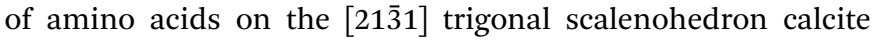
structure, which is the major component of limestone, has been highlighted. ${ }^{53,54}$ Calcite crystals possess intrinsic chiral selectivity on their surfaces due to their pairs of adjacent facets with mirror symmetry. Teng and co-workers demonstrated that the addition of aspartic acid dramatically affected the growth morphology of calcite, showing that the adsorbed layer aligned on the facets changes the surface energy. ${ }^{55}$ In the early $2000 \mathrm{~s}$, Hazen and co-workers reported an experimental demonstration of the selective adsorption of $\mathrm{D}^{-}$and $\mathrm{L}$-amino acids on calcite. ${ }^{53}$ Adjacent scalenohedral faces of calcite crystals equivalent to $(21 \overline{3} 1)$ and $(31 \overline{2} \overline{1})$ showed the selective adsorption of $D^{-}$and $\mathrm{L}$-aspartic acid up to $10 \%$ when immersed in the racemic aspartic acid solution, while the achiral surface showed no adsorption difference. This selective adsorption takes place preferentially on the kinks along the steps, indicating the preferential adsorption of one enantiomer on chiral terraced surfaces.

Enantio-specific binding of the amino acids could lead to macroscopic crystal shape modifications by changing the free energies of the step-edges, thereby leading to chiral deformation in macroscopic length scales. ${ }^{56}$ Chiral morphology development through the encoding of chiral biomolecules has been reported in several semiconducting materials such as cinnabar $(\alpha-\mathrm{HgS}),{ }^{6}$ potassium dichromate, ${ }^{57}$ selenium, and tellurium. ${ }^{58}$ These materials exhibit unique chiroptical properties due to their morphological chirality induced by the enantio-specific binding of biomolecules. The CD response of semiconducting materials solely originates from the chiral morphology, whereas the CD activity in metal nanostructures has origins in structural and plasmonic properties. ${ }^{58,59}$

Recently, Wang and coworkers demonstrated the chiral construction of cinnabar mercury sulfide nanocrystals with twisted shapes that originated from surface deformation by D- and L-penicillamine molecules (Fig. 4A). Cinnabar $\alpha-\mathrm{HgS}$ lattice with the space group of $P 3_{2} 21$ possesses an atomic scale primary chiral unit. In this demonstration, the handedness of the morphology from the epitaxial synthesis depends mainly on the type of chiral molecules used during the growth rather than the crystallographic chirality of the seed nanoparticles. For example, in the epitaxial synthesis, the initial seed crystallographic chirality is directly portrayed in the final morphology upon the addition of precursor atoms in the absence of chiral molecules. However, in the presence of chiral biomolecules, the handedness of chiral molecules determines the chirality of the atomic conformation on the surface. Consequently, morphology transfer occurs from handed biomolecules to the entire nanostructures on a larger scale. Ben-Moshe and coworkers reported chiral selenium and tellurium nanocrystals synthesized through the enantioselectively oriented adsorption
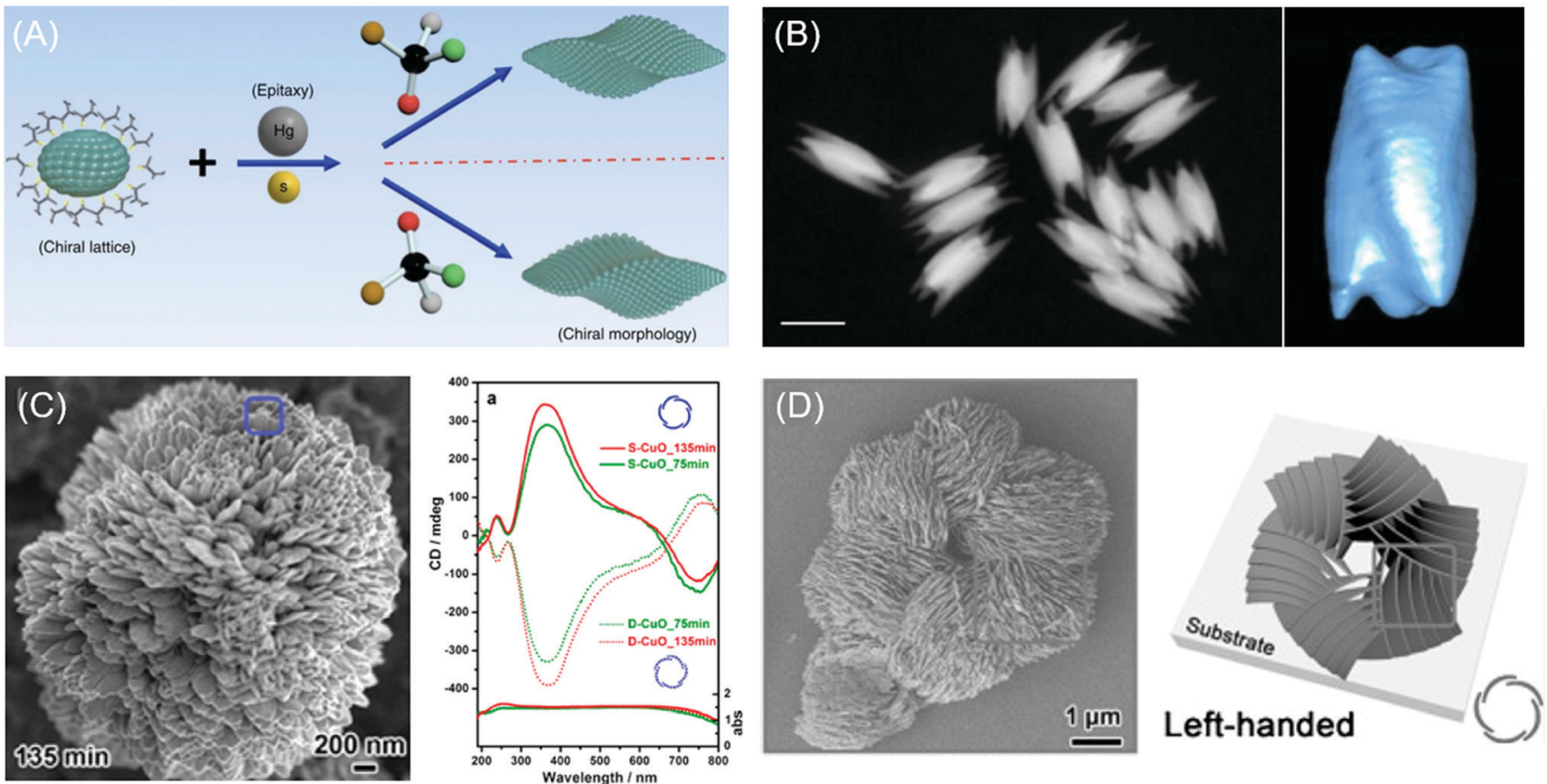

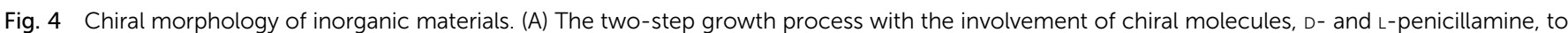

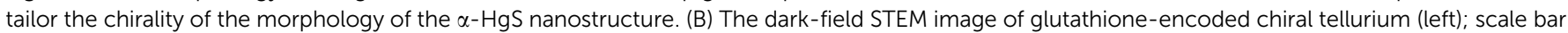

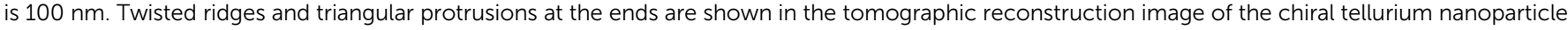

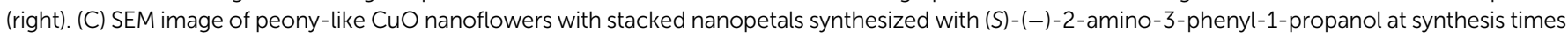

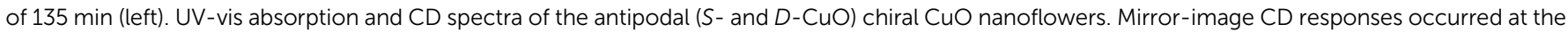

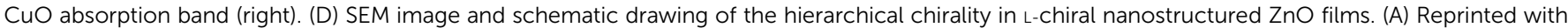

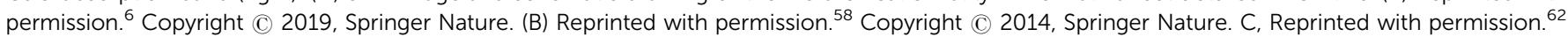
Copyright (C) 2014, American Chemical Society. D, Reprinted with permission. ${ }^{63}$ Copyright (C) 2015, WILEY-VCH. 
of chiral biomolecules. Chiral tellurium nanoparticles shown in Fig. 4B were synthesized in the presence of glutathione and possess twisted ridges and triangular protrusions at the ends. Using different biomolecules such as cysteine and penicillamine, different shapes of tellurium nanoparticles were obtained. Chiral nanocrystals are formed by the initial formation of chiral atomic clusters and the attachment of those clusters in an oriented ordering. The thiolate ligand molecule determines the geometry and stability of the Te complex, thereby affecting the growth kinetics.

Extending chiral structure generation from the atomic scale to the microscale is possible through the self-assembly of chiral molecules and inorganic materials. Bio-mineralization of nanoscale calcium carbonate building blocks in the presence of enantiomeric amino acids could induce large-scale chiral vaterite with counterclockwise or clockwise toroids. ${ }^{60,61} \mathrm{Jiang}$ and co-workers suggested that the tilting of subunits induced by attached chiral additives results in the cascade tilting of adjacent subunits, thus generating the macroscopic chiral structure. ${ }^{60}$ Also, the self-assembly of $\mathrm{CuO}^{62}$ and $\mathrm{ZnO}^{63}$ films with hierarchical chiral structures has been reported. Duan and coworkers reported the flower-shaped $\mathrm{CuO}$ nanostructure with hierarchical chirality using an amino alcohol as a symmetrybreaking agent. Nanopetals in the flower-shaped $\mathrm{CuO}$ nanostructure are composed of several sub-nanopetals that are stacked in a left- and right-handed helical structure. As expected, the flower-like $\mathrm{CuO}$ nanoparticles formed in the presence of amino alcohol with opposite chirality exhibited the same structure in the opposite direction (Fig. 4C). ${ }^{62}$ A year later, Wang and coworkers reported a hierarchical chiral superstructure of self-assembled $\mathrm{ZnO}$ film that exhibited large optical activity attributed to light scattering and absorption in the chiral hierarchical structures (Fig. 4D). The asymmetric attachment of $\mathrm{L}^{-}$and $\mathrm{D}-$ methionine with $\mathrm{Zn}^{2+}$ ions, which act as a symmetry-breaking agent, generates the chiral hierarchical $\mathrm{ZnO}$ nanostructure on a quartz substrate. ZnO films generated helical nanoplates as the primary chiral structure while the helical stacking of aggregates of primary units formed secondary and tertiary chiral structures. In the presence of symmetry-breaking molecules, ZnO nanoplates were stacked in the opposite direction of the handedness of the primary chiral unit due to the bending of the nanoplates. Microscale secondary and tertiary structure induced scattering-based CD signals, whereas absorption-based CD originated from the primary chirality of the helical structures in $\mathrm{ZnO}$ films.

\subsection{Interaction between peptide-based materials and plasmonic nanoparticles}

The interaction between peptide-based molecules and metal surfaces has been widely exploited within plasmonics and biosensing fields. The spontaneous attachment of cysteinecontaining peptide on metal surfaces, especially gold, shows strong interactions to form the $\mathrm{Au}-\mathrm{S}$ bond to provide the foundation for robust peptide-based structure fabrication. The amine group of peptides and DNA also adsorbs onto metal surfaces forming covalent bonds. The strong interactions between peptides and the metal surface and the well-defined self-assembled structure of the peptide enable the fabrication of the plasmonic nanoparticle structure using peptidebased material scaffolds. The spatial control of the individual plasmonic nanoparticles in the assembled macrostructure can be precisely controlled by utilizing programmable sequences of the peptide. Strong interactions of metal surfaces with peptides were demonstrated by assembling gold nanorods ${ }^{64-67}$ and nanospheres ${ }^{68,69}$ into macroscopic structures using amine groups, thiol groups, and electrostatic attraction. Lu and coworkers showed the important role of the strong Au-thiol bond between GSH molecules and gold nanorods, which resulted in the chiral assembled structure. ${ }^{66}$ The $\mathrm{L}^{-}$and $\mathrm{D}^{-G S H}$ ligands facilitate the end-to-end assembly of $\mathrm{Au}$ NRs in aqueous solution. In the aqueous solution, the cationic surfactant cetyltrimethylammonium bromide (CTAB) forms tight bilayers on the Au NR surfaces. In the presence of GSH molecules, GSH replaced CTAB at the ends of the NRs due to the relatively weaker interaction between CTAB and Au NR surfaces. Polar Glu residues facing outward undergo self-association to form oligomers, enabling the self-assembled chain structure.

With the aid of the binding motif, plasmonic nanoparticles could bind to even larger peptide-based scaffolds such as proteins, DNA, and viruses. ${ }^{68-78}$ For example, bacteriophages with the exposed Val-Ser-Gly-Ser-Ser-Pro-Asp-Ser (VSGSSPDS) $\mathrm{N}$-terminal sequence have been applied in the formation of gold nanoarchitectures based on its reactivity to gold surfaces. ${ }^{74,79}$ Recently, Lee and coworkers showed that peptides with the Pro-Asp (PD) sequence could provide strong binding of gold nanocubes. ${ }^{73}$ A series of bacteriophage libraries with randomized exposed $\mathrm{N}$-terminal amino acid motifs were found by experiment to have an optimal binding affinity for gold nanoparticles and efficient bacteriophage amplification. The Pro-Asp sequence exhibited amplification yields and the optimal binding of gold nanocubes, suggesting that the Pro-Asp sequence possesses or can increase the affinity for gold nanoparticles. Using the virus as a structural scaffold, the precise modification of protein at the peptide level in the most exposed capsid layer allowed a strong binding affinity for the gold surface and the fabrication of hybrid gold-cobalt oxide nanowires. ${ }^{70}$ The virus scaffolds were utilized to assemble plasmonic gold nanocubes into closely aligned chains along the virus, resulting in enhanced Raman signals with the consequence of increased local electric field. ${ }^{71}$

\subsection{Chiral morphology evolution in plasmonic materials}

Chirality transfer from molecular structure to macroscopic crystals could be expanded to plasmonic materials in the nanoscale. $^{21,80-83}$ The preferential attachment of the biomolecules enabled morphology modulation of metal surfaces in a single particle level, whereas earlier studies focused on the chiral structure of self-assembled plasmonic nanoparticles and the chiroptical responses. ${ }^{84-89}$ Due to the plasmonic properties, single plasmonic nanoparticles with chiral morphology could induce strong chiroptical responses at its plasmon frequency. Based on the Drude model, Fan and co-workers conducted a 
computational modal analysis of plasmon resonances of a chiral nanocrystal and showed that a chiral twister with helical surface exhibited strong CD responses at the main plasmonic resonance. Upon adsorption of chiral molecules on the surface, the crystal surface with a low Miller index undergoes reconstruction to expose chiral high Miller index planes. Generated chiral distortions of plasmonic nanocrystal create splitting and mixing of plasmonic modes, inducing strong optical chirality.

Recently, our group experimentally demonstrated the exceptional chiroptical response of chemically synthesized chiral gold nanoparticles at the $100 \mathrm{~nm}$ scale using cysteine and cysteine-containing peptides as chiral shape modifiers. ${ }^{90}$ With respect to the chirality of the chiral shape modifier, the
(A)

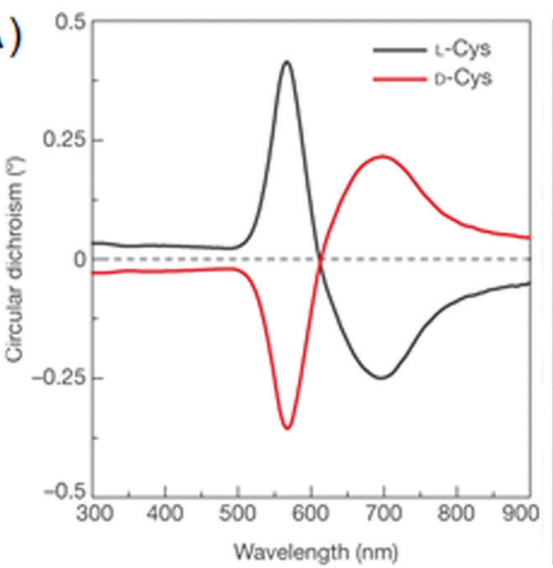

(B)
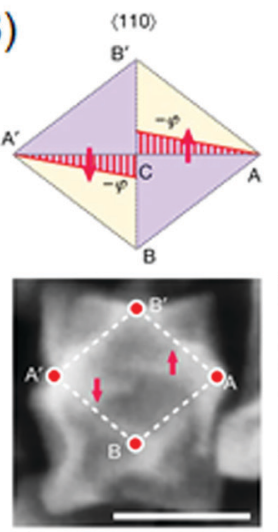
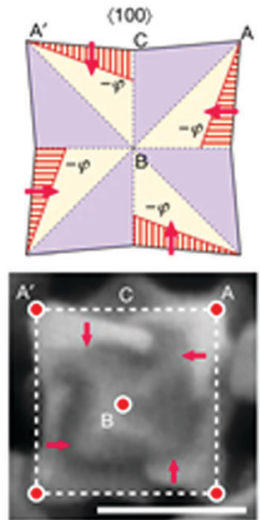
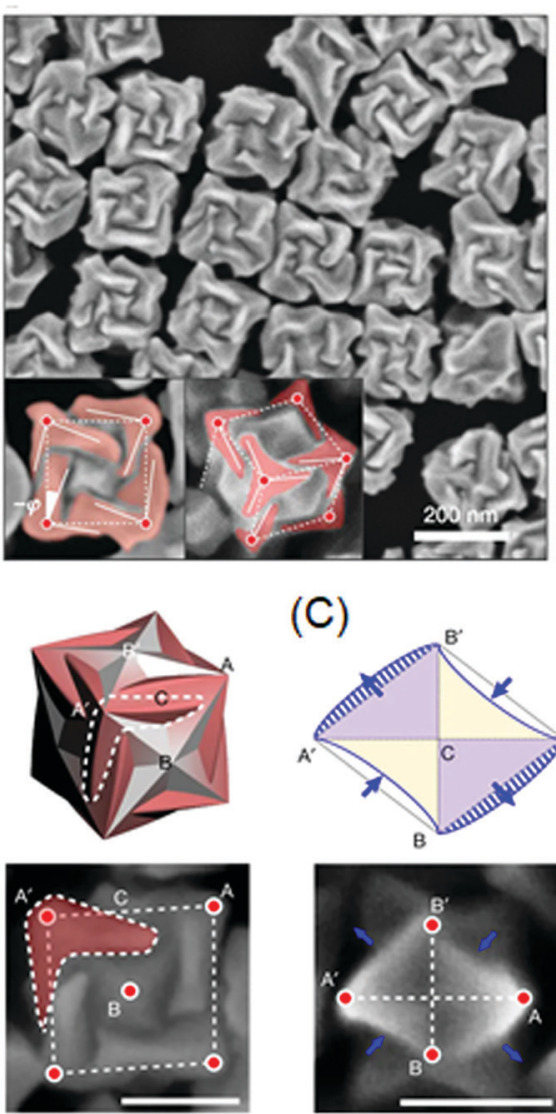

(C)
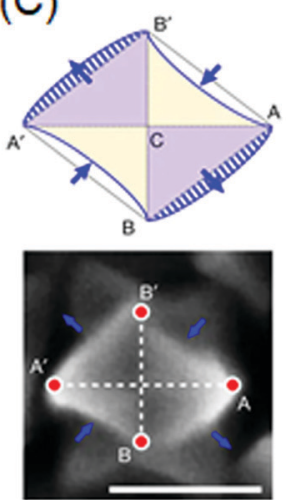
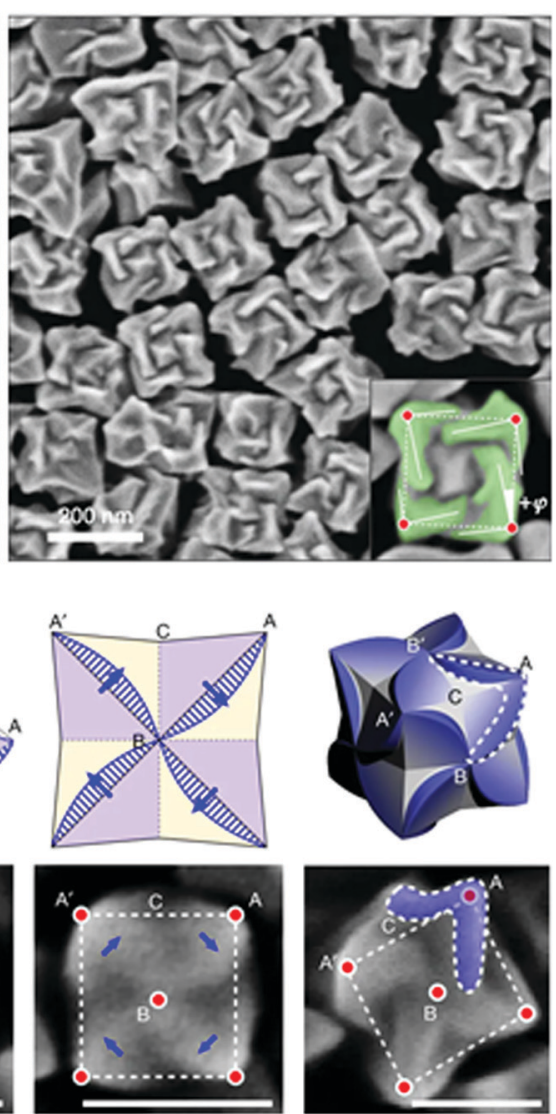

(D)

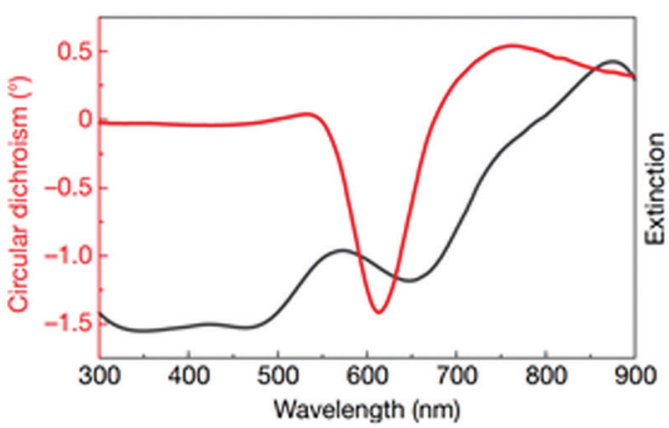

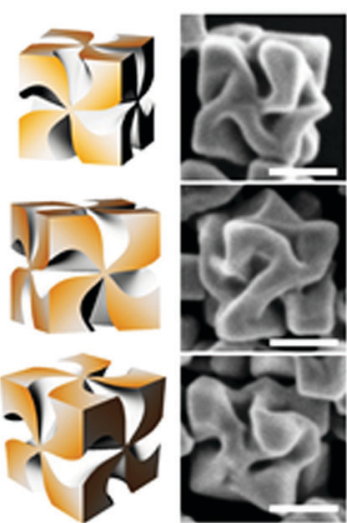

(E)

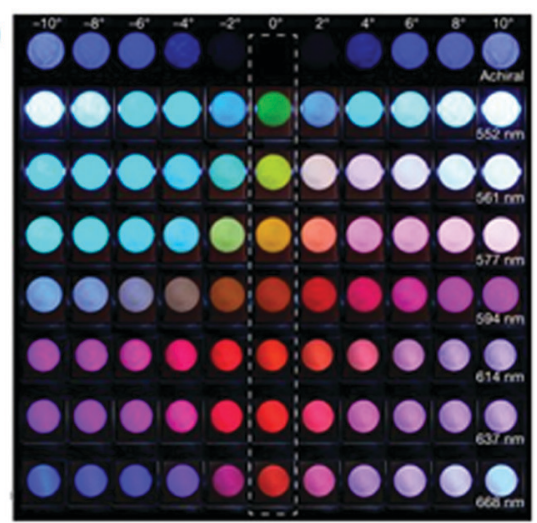

Fig. 5 Chiral morphology of plasmonic gold nanoparticles. (A) CD spectra of 432 helicoid I synthesized with L-cysteine (black line) and D-cysteine (red line) (left). SEM images of 432 helicoid I synthesized with L-cysteine (middle). SEM images of 432 helicoid I synthesized with D-cysteine (right). (B) Schematics and SEM images of the morphology evolution of the chiral helicoid in the presence of L-cysteine, as viewed along the [110] direction (left), the [100] direction (middle), and the final 3D morphology (right). The scale bar is $100 \mathrm{~nm}$. (C) Schematics and SEM images of the morphology evolution of the chiral helicoid in the presence of L-GSH as viewed along the [110] direction (left), [100] direction (middle), and the final 3D morphology (right). The scale bar is $100 \mathrm{~nm}$. (D) Circular dichroism and extinction spectra (left), and 3D models and corresponding SEM images of 432 helicoid III (right). The scale bar is $100 \mathrm{~nm}$. (E) The transmitted color of achiral particles and 432 helicoid III particle solutions with different sizes under cross-polarized conditions (dashed box). Gradual color tuning was observed by rotating the analyzer from $-10^{\circ}$ to $10^{\circ}$. Reprinted with permission..$^{90}$ Copyright (C) 2018, Springer Nature. 
synthesized nanoparticles show mirror symmetry in the nanomorphology and the chiroptical response also shows perfect mirror symmetry with absorption peaks at $569 \mathrm{~nm}$ (Fig. 5A). As stated above, surface chirality can exist even for highly symmetric metal crystals faces that lack mirror symmetry. All $\{h k l\}(h \neq k \neq l \neq 0)$ high-Miller-index surfaces are nonsuperimposable and are denoted as $R(S)$, depending on its ordering of low-index crystal planes. This intrinsic chirality of crystal planes enables enantioselective interaction between molecules to form plasmonic inorganic materials. Therefore, it is crucial to provide chiral atomic sites for the successive transfer of molecular chirality to plasmonic materials. For precise control of exposed high-index facets, seed-mediated synthesis using an organothiol additive as the shape modifier was implemented. ${ }^{91-96}$ With the addition of chiral additives, the enantioselective interaction between the L-peptide with the generated high-Miller-index facet shifts the boundaries of adjacent facets with different chirality as growth proceeds. This asymmetric growth results in the breaking of mirror and inversion symmetry to generate the 432-point group symmetry, which is therefore named the 432 helicoid series. In the case of the L-cysteine molecule, $\mathrm{A}^{\prime} \mathrm{C}$ and $\mathrm{AC}$ edges viewed from the (110) direction shifts in the opposite direction. More clearly, viewed from the (100) direction, the outer edges of nanoparticle $\mathrm{A}^{\prime} \mathrm{C}$ and $\mathrm{AC}$ shift towards the center of the nanoparticle in a clockwise rotation to generate chiral morphology while the input of $\mathrm{D}$-cysteine results in the counterclockwise rotation of edges (Fig. 5B).

Interestingly, the change in the chiral molecule from cysteine to the cysteine-containing glutathione (GSH) molecule induced a different chiral morphology. As shown in Fig. 5C, growth after the addition of the L-GSH molecule induced the contraction of the $\mathrm{AB}^{\prime}$ and $\mathrm{A}^{\prime} \mathrm{B}$ edges and the protrusion of the $A^{\prime} B^{\prime}$ and $A B$ edges when viewed from the (110) direction. From the (100) direction, it is possible to observe the clockwise rotation of the inner edges $\mathrm{A}^{\prime} \mathrm{B}$ and $\mathrm{AB}$ to form a pinwheellike structure. Further tuning of the chiral morphology and the chiroptical response was possible through the control of the seed morphology. On using the GSH molecule and the $\{111\}$ facet-enclosed gold octahedron seed, denoted as 432 helicoid III, chiral nanoparticles containing four high-curved gaps on each of the six facets of the cubic geometry with pinwheellike structures were generated (Fig. 5D). The 432 helicoid III nanoparticles exhibited outstanding chiroptical activity with an asymmetry factor of 0.2. Utilizing this high chiroptical response, direct visualizations of macroscopic color conversions under polarization conditions are depicted in Fig. 5E. This exceptional chiroptical response could be attributed to the highly twisted arms and gaps as compared to other helicoid morphologies, which is supported by the microscopic image of the He-ion milled helicoid III nanoparticles.

This bottom-up synthetic route using biomolecules to generate chirality provides a promising pathway for versatility in morphology control. There are still many possibilities with biomolecule-directed plasmonic chiral structures with exceptional chiroptical activity. For example, numerous chiral peptide structures are readily available, which show multicomplexity in molecular structures as displayed in Table 1. By harnessing the sophisticated chiral structures of peptide-based materials and their tendency for specific interaction with nanomaterials, numerous biomolecules such as peptides, proteins, DNA, and antibodies can be exploited as chiral shape modifiers to develop unique and novel chiral morphologies. In this respect, this novel chemical synthetic route for chirality evolution can provide a new paradigm for optical devices such as chiral sensors, active color displays, holography, and negative refractive index materials.

\section{Conclusions}

In this review, we start by exploiting recent investigations to identify exceptional roles of amino acids and peptides in chirality, from local atomic conformation to macroscopic chiral morphology. Inorganic materials could possess chiral space groups and even some achiral crystals could possess a chiral surface due to the absence of mirror symmetry. These chiral atomic structures create an asymmetric environment for biomolecules, which provides an excellent platform for the study of symmetry. In this regard, the conjugation of chiral molecules with inorganic surfaces allows for closer interplay at the interface between biomolecules and the inorganic surface. These interactions induce permanent distortions or reconstructions at the atomic sites, which could display chiroptical responses originating from the material itself.

The exceptional abilities of amino acids and peptides to induce the reconstruction of inorganic interfaces could be extended to the three-dimensional chiral morphology of

Table 1 Data are from previous work ${ }^{97-104}$

\begin{tabular}{|c|c|c|c|}
\hline Materials & & Structure & Ref. \\
\hline \multirow[t]{2}{*}{ Peptide } & $\mathrm{E} 5, \mathrm{FlgA}_{3}$ & Au NP coated with Peptide & 97 \\
\hline & $\mathrm{PEG}^{-\mathrm{NH}_{2}}$, PEG-OMe & Twisted Au nanorod oligomer & 98 \\
\hline \multirow[t]{4}{*}{ DNA } & Thiolated DNA & Au NP tetrahedral superstructure & 99 \\
\hline & Thiolated DNA & DNA origami bundle & 100 \\
\hline & DNA & Bifacial DNA origami bundle & 101 \\
\hline & DNA & Twisted Au nanorod dimer & 102 \\
\hline Antibody & $\mathrm{mAb}, \mathrm{smAb}$ & $\mathrm{Au}-\mathrm{Ag} \mathrm{NP}$ heterodimer & 103 \\
\hline Chiral supromolecule & Chiral molecular ligand & Ag NP coated with assembled chiral supramolecule & 104 \\
\hline
\end{tabular}


inorganic nanostructures. From a single nanoparticle perspective, enantiospecific interactions between chiral molecules and chiral atomic sites induce chiral nano-morphology evolution. Further control of the interface chemistry generates macroscopic hierarchical chiral structures. With the use of plasmonic material, this sophisticated and highly controlled morphology generates an exceptionally strong chiroptical response at the plasmonic resonance. Based on the understanding of organicinorganic interactions, the encoding of amino acids and peptides onto inorganic structures shows a very promising pathway in current molecular science with unlimited variations in molecular structure. Therefore, in order to meet the requirements of practical application, we envision that amino acid and peptide-based chiral morphology evolution can play a pivotal role in many practical applications related to chirality, such as chiral optical devices, chiral selective sensor and chirality separation filters.

\section{Conflicts of interest}

There are no conflicts to declare.

\section{Acknowledgements}

This work was supported by the KIST-SNU Joint Research Lab project (2V06170) under the KIST Institutional Program funded by the Korea government (Ministry of Science, ICT \& Future Planning). This work was supported by the National Research Foundation of Korea (NRF) grant funded by the Korea government (MSIT) (NRF-2017R1A2B3012003). KTN appreciates the support from Institute of Engineering Research, Research Institute of Advanced Materials (RIAM) and Soft Foundry at Seoul National University.

\section{Notes and references}

1 L. Pasteur, Ann. Chim. Phys., 1848, 24, 442-459.

2 M. Schilthuizen and A. Davison, Naturwissenschaften, 2005, 92, 504-515.

3 G. E. Schröder-Turk, S. Wickham, H. Averdunk, F. Brink, J. D. Fitz Gerald, L. Poladian, M. C. J. Large and S. T. Hyde, J. Struct. Biol., 2011, 174, 290-295.

4 J. Lekner, Pure Appl. Opt. J. Eur. Opt. Soc. Part A, 1996, 5, 417-443.

5 A. M. Glazer and K. Stadnicka, J. Appl. Crystallogr., 1986, 19, 108-122.

6 P. Wang, S.-J. Yu, A. O. Govorov and M. Ouyang, Nat. Commun., 2017, 8, 14312.

7 R. M. Hazen, Progress in Biological Chirality, Elsevier, 2004, pp. 137-151.

8 J. D. Horvath and A. J. Gellman, Top. Catal., 2003, 25, 9-15.

9 R. E. Gibbs, Proc. R. Soc. A, 2006, 110, 443-455.

10 R. M. Hazen and D. S. Sholl, Nat. Mater., 2003, 2, 367-374.

11 C. F. McFadden, P. S. Cremer and A. J. Gellman, Langmuir, 1996, 12, 2483-2487.
12 A. Ahmadi, G. Attard, J. Feliu and A. Rodes, Langmuir, 1999, 15, 2420-2424.

13 M. V. Mukhina, V. G. Maslov, A. V. Baranov, A. V. Fedorov, A. O. Orlova, F. Purcell-Milton, J. Govan and Y. K. Gun'Ko, Nano Lett., 2015, 15, 2844-2851.

14 I. Weissbuch and M. Lahav, Chem. Rev., 2011, 111, 3236-3267.

15 S. M. Barlow and R. Raval, Curr. Opin. Colloid Interface Sci., 2008, 13, 65-73.

16 X. Zhao, R. G. Zhao and W. S. Yang, Langmuir, 2000, 16, 9812-9818.

17 Y. Zhou, M. Yang, K. Sun, Z. Tang and N. A. Kotov, J. Am. Chem. Soc., 2010, 132, 6006-6013.

18 J. Kuno, Y. Imamura, M. Katouda, M. Tashiro, T. Kawai and T. Nakashima, Angew. Chem., Int. Ed., 2018, 57, 12022-12026.

19 A. Ben-Moshe, A. O. Govorov and G. Markovich, Angew. Chem., Int. Ed., 2013, 52, 1275-1279.

20 Y. Xia, Y. Zhou and Z. Tang, Nanoscale, 2011, 3, 1374-1382.

21 A. Ben-Moshe, B. M. Maoz, A. O. Govorov and G. Markovich, Chem. Soc. Rev., 2013, 42, 7028-7041.

22 J. B. Pendry, Science, 2004, 306, 1353-1355.

23 Z. Wang, F. Cheng, T. Winsor and Y. Liu, Nanotechnology, 2016, 27, 412001.

24 P. Shekhar, J. Atkinson and Z. Jacob, Nano Convergence, 2014, 1, 14.

25 S. S. Oh and O. Hess, Nano Convergence, 2015, 2, 24.

26 A. O. Govorov, Z. Fan, P. Hernandez, J. M. Slocik and R. R. Naik, Nano Lett., 2010, 10, 1374-1382.

27 T. G. Schaaff, G. Knight, M. N. Shafigullin, R. F. Borkman and R. L. Whetten, J. Phys. Chem. B, 2002, 102, 10643-10646.

28 H. Yao, T. Fukui and K. Kimura, J. Phys. Chem. C, 2007, 111, 14968-14976.

29 H. Chang, Y. Y. Lee, H. E. Lee, H. Y. Ahn, E. Ko, K. T. Nam and D. H. Jeong, Phys. Chem. Chem. Phys., 2019, 21, 9044-9051.

30 C. Gautier and T. Bürgi, J. Am. Chem. Soc., 2008, 130, 7077-7084.

31 M. C. Di Gregorio, A. Ben Moshe, E. Tirosh, L. Galantini and G. Markovich, J. Phys. Chem. C, 2015, 119, 17111-17116.

32 T. Levi-Belenkova, A. O. Govorov and G. Markovich, J. Phys. Chem. C, 2016, 120, 12751-12756.

33 U. Tohgha, K. Varga and M. Balaz, Chem. Commun., 2013, 49, 1844-1846.

34 X. Gao, X. Zhang, L. Zhao, P. Huang, B. Han, J. Lv, X. Qiu, S. H. Wei and Z. Tang, Nano Lett., 2018, 18, 6665-6671.

35 U. Tohgha, K. K. Deol, A. G. Porter, S. G. Bartko, J. K. Choi, B. M. Leonard, K. Varga, J. Kubelka, G. Muller and M. Balaz, ACS Nano, 2013, 7, 11094-11102.

36 J. Cheng, J. Hao, H. Liu, J. Li, J. Li, X. Zhu, X. Lin, K. Wang and T. He, ACS Nano, 2018, 12, 5341-5350.

37 A. Ben-Moshe, A. Teitelboim, D. Oron and G. Markovich, Nano Lett., 2016, 16, 7467-7473.

38 A. Ben Moshe, D. Szwarcman and G. Markovich, ACS Nano, 2011, 5, 9034-9043.

39 M. Naito, K. Iwahori, A. Miura, M. Yamane and I. Yamashita, Angew. Chem., Int. Ed., 2010, 49, 7006-7009. 
40 Y. Zhou, Z. Zhu, W. Huang, W. Liu, S. Wu, X. Liu, Y. Gao, W. Zhang and Z. Tang, Angew. Chem., Int. Ed., 2011, 50, 11456-11459.

41 X. Gao, X. Zhang, K. Deng, B. Han, L. Zhao, M. Wu, L. Shi, J. Lv and Z. Tang, J. Am. Chem. Soc., 2017, 139, 8734-8739.

42 G. Yang, M. Kazes and D. Oron, Adv. Funct. Mater., 2018, 28, 1-9.

43 P. D. Jadzinsky, G. Calero, C. J. Ackerson, D. A. Bushnell and R. D. Kornberg, Science, 2007, 318, 430-433.

44 T. G. Schaaff and R. L. Whetten, J. Phys. Chem. B, 2002, 104, 2630-2641.

45 M. P. Moloney, Y. K. Gun'ko and J. M. Kelly, Chem. Commun., 2007, 3900-3902.

46 T. Nakashima, Y. Kobayashi and T. Kawai, J. Am. Chem. Soc., 2009, 131, 10342-10343.

47 M. V. Mukhina, I. V. Korsakov, V. G. Maslov, F. PurcellMilton, J. Govan, A. V. Baranov, A. V. Fedorov and Y. K. Gun'ko, Sci. Rep., 2016, 6, 24177.

48 S. Jiang, M. Chekini, Z. B. Qu, Y. Wang, A. Yeltik, Y. Liu, A. Kotlyar, T. Zhang, B. Li, H. V. Demir and N. A. Kotov, J. Am. Chem. Soc., 2017, 139, 13701-13712.

49 J. Yeom, U. S. Santos, M. Chekini, M. Cha, A. F. De Moura and N. A. Kotov, Science, 2018, 359, 309-314.

50 T. Tanaka and A. Ishikawa, Nano Convergence, 2017, 4, 34.

51 C. Cai, J. Lin, Y. Lu, Q. Zhang and L. Wang, Chem. Soc. Rev., 2016, 45, 5985-6012.

52 Y. Wang, J. Xu, Y. Wang and H. Chen, Chem. Soc. Rev., 2013, 42, 2930-2962.

53 R. M. Hazen, T. R. Filley and G. A. Goodfriend, Proc. Natl. Acad. Sci. U. S. A., 2001, 98, 5487-5490.

54 W. E. F. E. S. Dana, A Textbook of Mineralogy, Wiley, New York, 4th edn, 1958.

55 H. H. Teng, P. M. Dove, C. A. Orme and J. J. D. Yoreo, Science, 1998, 282, 724-727.

56 C. A. Orme, A. Noy, A. Wierzbicki, M. T. McBride, M. Grantham, H. H. Teng, P. M. Dove and J. J. DeYoreo, Nature, 2001, 411, 775-779.

57 H. I. Yuya Oaki, J. Am. Chem. Soc., 2004, 126, 9271-9275.

58 A. Ben-Moshe, S. G. Wolf, M. B. Sadan, L. Houben, Z. Fan, A. O. Govorov and G. Markovich, Nat. Commun., 2014, 5, 4302.

59 Z. Fan and A. O. Govorov, Nano Lett., 2012, 12, 3283-3289.

60 W. Jiang, M. S. Pacella, D. Athanasiadou, V. Nelea, H. Vali, R. M. Hazen, J. J. Gray and M. D. McKee, Nat. Commun., 2017, 8, 15066.

61 W. Jiang, M. S. Pacella, H. Vali, J. J. Gray and M. D. McKee, Sci. Adv., 2018, 4, eaas9819.

62 Y. Duan, X. Liu, L. Han, S. Asahina, D. Xu, Y. Cao, Y. Yao and S. Che, J. Am. Chem. Soc., 2014, 136, 7193-7196.

63 Y. Duan, L. Han, J. Zhang, S. Asahina, Z. Huang, L. Shi, B. Wang, Y. Cao, Y. Yao, L. Ma, C. Wang, R. K. Dukor, L. Sun, C. Jiang, Z. Tang, L. A. Nafie and S. Che, Angew. Chem., Int. Ed., 2015, 54, 15170-15175.

64 Z. Zhu, W. Liu, Z. Li, B. Han, Y. Zhou, Y. Gao and Z. Tang, ACS Nano, 2012, 6, 2326-2332.

65 F. Zhu, X. Li, Y. Li, M. Yan and S. Liu, Anal. Chem., 2015, 87, 357-361.
66 J. Lu, Y.-X. Chang, N.-N. Zhang, Y. Wei, A.-J. Li, J. Tai, Y. Xue, Z.-Y. Wang, Y. Yang, L. Zhao, Z.-Y. Lu and K. Liu, ACS Nano, 2017, 11, 3463-3475.

67 B. Han, Z. Zhu, Z. Li, W. Zhang and Z. Tang, J. Am. Chem. Soc., 2014, 136, 16104-16107.

68 X. Fu, Y. Wang, L. Huang, Y. Sha, L. Gui, L. Lai and Y. Tang, Adv. Mater., 2003, 15, 902-906.

69 G. Grigoryan, Y. H. Kim, R. Acharya, K. Axelrod, R. M. Jain, L. Willis, M. Drndic, J. M. Kikkawa and W. F. DeGrado, Science, 2011, 332, 1071-1076.

70 K. T. Nam, D. W. Kim, P. J. Yoo, C. Y. Chiang, N. Meethong, P. T. Hammond, Y.-M. Chiang and A. M. Belcher, Science, 2006, 312, 885-888.

71 H.-E. Lee, H. K. Lee, H. Chang, H.-Y. Ahn, N. Erdene, H.-Y. Lee, Y.-S. Lee, D. H. Jeong, J. Chung and K. T. Nam, Small, 2014, 10, 3007-3011.

72 C. Song, M. G. Blaber, G. Zhao, P. Zhang, H. C. Fry, G. C. Schatz and N. L. Rosi, Nano Lett., 2013, 13, 3256-3261.

73 H. K. Lee, Y. Lee, H. Kim, H.-E. Lee, H. Chang, K. T. Nam, D. H. Jeong and J. Chung, ACS Synth. Biol., 2017, 6, 1635-1641.

74 Y. Huang, C.-Y. Chiang, S. K. Lee, Y. Gao, E. L. Hu, J. De Yoreo and A. M. Belcher, Nano Lett., 2005, 5, 1429-1434.

75 K. L. Gurunatha, A. C. Fournier, A. Urvoas, M. ValerioLepiniec, V. Marchi, P. Minard and E. Dujardin, ACS Nano, 2016, 10, 3176-3185.

76 D. M. Raymond and B. L. Nilsson, Chem. Soc. Rev., 2018, 47, 3659-3720.

77 S. Zhang, Nat. Biotechnol., 2003, 21, 1171-1178.

78 C.-L. Chen, P. Zhang and N. L. Rosi, J. Am. Chem. Soc., 2008, 130, 13555-13557.

79 Y. J. Lee, Y. Lee, D. Oh, T. Chen, G. Ceder and A. M. Belcher, Nano Lett., 2010, 10, 2433-2440.

80 N. H. Cho, H. Lee, H. Ahn, Y. Y. Lee, S. W. Im, H. Kim and K. T. Nam, Part. Part. Syst. Charact., 2019, 36, 1900062.

81 H.-E. Lee, J. Lee, M. Ju, H.-Y. Ahn, Y. Y. Lee, H.-S. Jang and K. T. Nam, Mol. Syst. Des. Eng., 2018, 3, 581-590.

82 X. T. Kong, L. V. Besteiro, Z. Wang and A. O. Govorov, $A d v$. Mater., 2018, 1801790, 1-14.

83 J. Yan, Y. Chen, S. Hou, J. Chen, D. Meng, H. Zhang, H. Fan, Y. Ji and X. Wu, Nanoscale, 2017, 9, 11093-11102.

84 J. Cheng, G. Le Saux, J. Gao, T. Buffeteau, Y. Battie, P. Barois, V. Ponsinet, M. H. Delville, O. Ersen, E. Pouget and R. Oda, ACS Nano, 2017, 11, 3806-3818.

85 B. M. Maoz, R. Van Der Weegen, Z. Fan, A. O. Govorov, G. Ellestad, N. Berova, E. W. Meijer and G. Markovich, J. Am. Chem. Soc., 2012, 134, 17807-17813.

86 G. Shemer, O. Krichevski, G. Markovich, T. Molotsky, I. Lubitz and A. B. Kotlyar, J. Am. Chem. Soc., 2006, 128, 11006-11007.

87 K. Hirai, B. Yeom and K. Sada, ACS Nano, 2017, 11, 5309-5317.

88 H.-E. Lee, H.-Y. Ahn, J. Lee and K. T. Nam, ChemNanoMat, 2017, 3, 685-697.

89 T. Nagamune, Nano Convergence, 2017, 4, 9.

90 H.-E. Lee, H.-Y. Ahn, J. Mun, Y. Y. Lee, M. Kim, N. H. Cho, K. Chang, W. S. Kim, J. Rho and K. T. Nam, Nature, 2018, 556, 360-365. 
91 H.-Y. Ahn, H.-E. Lee, K. Jin and K. T. Nam, J. Mater. Chem. C, 2013, 1, 6861.

92 M. A. Fuller and I. Köper, Nano Convergence, 2019, 6, 11.

93 H.-E. Lee, K. D. Yang, S. M. Yoon, H.-Y. Ahn, Y. Y. Lee, H. Chang, D. H. Jeong, Y.-S. Lee, M. Y. Kim and K. T. Nam, ACS Nano, 2015, 9, 8384-8393.

94 Y. J. Kang, E. G. Cutler and H. Cho, Nano Convergence, 2018, 5, 35.

95 H. Chang, Y. Y. Lee, H. E. Lee, H.-Y. Ahn, E. Ko, K. T. Nam and D. H. Jeong, Phys. Chem. Chem. Phys., 2019, 21, 9044-9051.

96 H.-E. Lee, R. M. Kim, H.-Y. Ahn, Y. Y. Lee, G. H. Byun, S. W. Im, J. Mun, J. Rho and K. T. Nam, Nat. Commun., 2020, 11, 263.

97 J. M. Slocik, A. O. Govorov and R. R. Naik, Nano Lett., 2011, 11, 701-705.
98 J. Yan, S. Hou, Y. Ji and X. Wu, Nanoscale, 2016, 8, 10030-10034.

99 W. Yan, L. Xu, C. Xu, W. Ma, H. Kuang, L. Wang and N. A. Kotov, J. Am. Chem. Soc., 2012, 134, 15114-15121.

100 A. Kuzyk, R. Schreiber, Z. Fan, G. Pardatscher, E.-M. Roller, A. Högele, F. C. Simmel, A. O. Govorov and T. Liedl, Nature, 2012, 483, 311-314.

101 X. Lan, X. Lu, C. Shen, Y. Ke, W. Ni and Q. Wang, J. Am. Chem. Soc., 2015, 137, 457-462.

102 A. Kuzyk, R. Schreiber, H. Zhang, A. O. Govorov, T. Liedl and N. Liu, Nat. Mater., 2014, 13, 862-866.

103 X. Wu, L. Xu, L. Liu, W. Ma, H. Yin, H. Kuang, L. Wang, C. Xu and N. A. Kotov, J. Am. Chem. Soc., 2013, 135, 18629-18636.

104 B. M. Maoz, R. van der Weegen, Z. Fan, A. O. Govorov, G. Ellestad, N. Berova, E. W. Meijer and G. Markovich, J. Am. Chem. Soc., 2012, 134, 17807-17813. 